\title{
LMI Approach to Robust Control of Rotary Cranes under Load Sway Frequency Variance*
}

\author{
Shigenori SANO**, Huimin OUYANG ${ }^{* *}$, Hiroshi YAMASHITA** and \\ Naoki UCHIYAMA** \\ ** Department of Mechanical Engineering, Toyohashi University of Technology \\ 1-1 Hibarigaoka, Tempaku, Toyohashi, Aichi, 441-8580, Japan \\ E-mail: sano@me.tut.ac.jp, ouyang_star007@yahoo.co.jp, uchiyama@tut.jp
}

\begin{abstract}
Because horizontal motion of a rotary crane generates undesirable two-dimensional load sway, skillful operators are needed to control the crane's motion; for this purpose, various types of control schemes have been proposed. Because natural frequency of the rope-load oscillation system affects the stability and performance of the control system, the controller design should consider robustness with respect to rope length. If the control system considers the effect of rope length variance, the crane's motion can be controlled without a sensor system for measuring it. This paper presents a control method based on linear matrix inequality optimization for achieving robustness with respect to rope length variance. Numerical simulations and experimental results demonstrate the effectiveness of the proposed method.
\end{abstract}

Key words : Rotary Crane, Motion Control, Robust Control, State Feedback Control with Integrator, LMI

\section{Introduction}

Crane systems are widely used in industrial applications, such as construction sites, ports, railway yards, and other locations. A crane system should be controlled for rapid transport of load without excessive load sway at the final position. However, one-dimensional horizontal boom motion of a rotary crane typically generates undesirable two-dimensional load sway; therefore, crane operators must be highly skilled in order to manually stop the load sway immediately at the right position. Failures of crane control cause accidents, injure people, and damage surroundings.

To reduce the burden on human operators and increase their safety, various control schemes have been developed for crane control. These methods can be classified into two types: trajectory generation methods for the crane's motion that can suppress load sway without requiring load sway information (i.e., open-loop control) ${ }^{(1)}{ }_{-}^{(9)}$ and methods using load sway information measured by sensor systems in real time to suppress load sway (i.e., closedloop control) ${ }^{(10)}{ }^{(13)}$. The former methods are valid only for an ideal system without disturbances such as wind; therefore, they may not achieve good outdoor performance. In contrast, the latter methods may be used to construct a robust control system for cranes.

However, existing studies do not consider robustness with respect to variations in rope length. If the control system considers the effect of rope length variance, the crane's motion can be controlled without a sensor system for measuring it. This study aims to propose a method to achieve this.

Because the control system is expected to be robust with respect to varying parameters such as joint friction, we first consider a controller design on the basis of a disturbance observer to provide the linear dynamics of rotary crane motion. Next, we design a state feedback controller with an integrator to achieve robust control performance for a given range of variations in rope length. The proposed control system satisfies additional constraint conditions

*Received 26 July, 2011 (No. 11-0457) [DOI: 10.1299/jsdd.5.1402]

Copyright (c) 2011 by JSME 


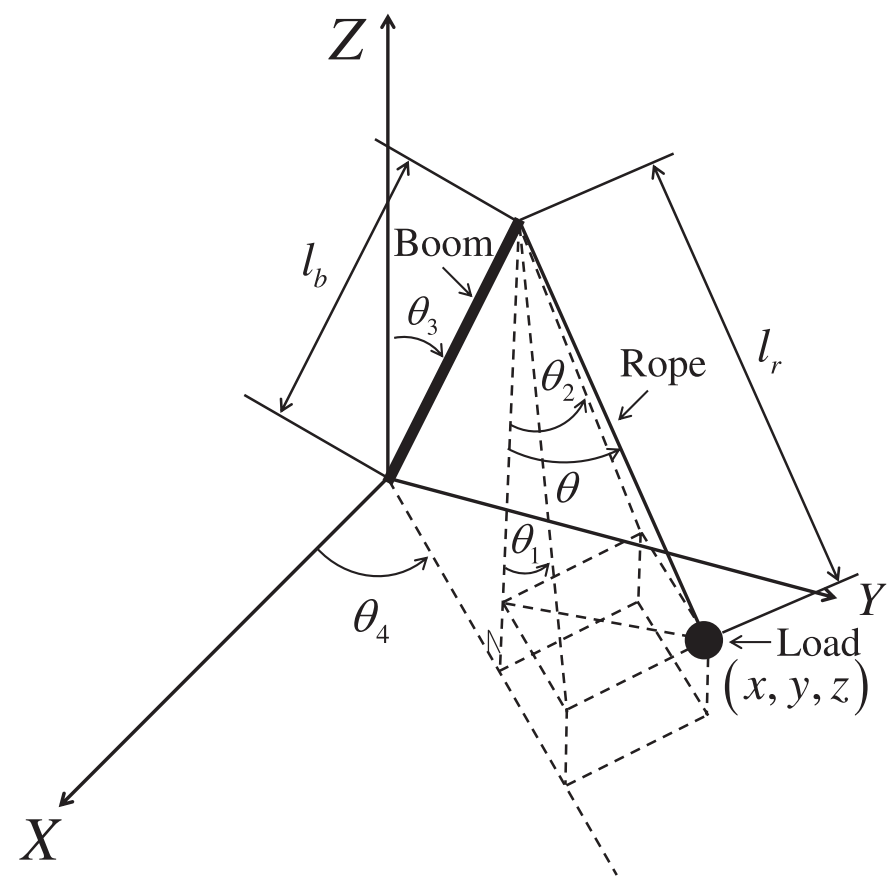

Fig. 1 Schematic model of rotary crane

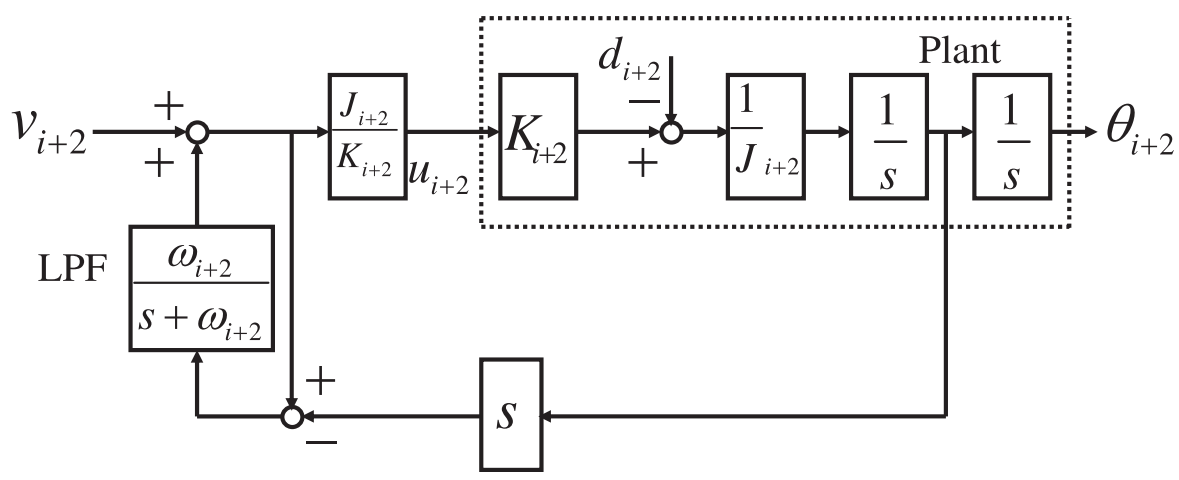

Fig. 2 Control system with disturbance observer

on closed-loop pole placement. These conditions are expressed in terms of linear matrix inequalities $(\mathrm{LMI})^{(14)}$, and controller gains are determined numerically via LMI optimization. Finally, simulations and experimental results demonstrate the effectiveness of the proposed method.

\section{Rotary Crane Dynamics}

In this section, we describe the dynamics of the rotary crane whose schematic model is shown in Fig. $1^{(13)}$. $\theta_{1}$ and $\theta_{2}$ are the load sway angles in the plane of vertical boom motion and the tangential direction of horizontal boom motion, respectively. $\theta_{3}$ and $\theta_{4}$ are the vertical and horizontal angles of the boom, respectively; $l_{r}$ and $l_{b}$ denote the lengths of the rope and boom, respectively; and $(x, y, z)$ denotes the three-dimensional position of the load.

We assume that the rotary crane dynamics has the following characteristics:

- The load can be considered to be a point mass, and the torsion of the rope can be neglected.

- The angle and angular velocity $\left(\theta_{i+2}\right.$ and $\dot{\theta}_{i+2}, i=1,2$, respectively) of vertical and horizontal boom motion and the load sway angles and their angular velocities $\left(\theta_{i}\right.$ and $\dot{\theta}_{i}, i=$ 
1,2 , respectively) are measurable.

- The sway angle $\theta_{i}$ has a small magnitude, so that approximations $\sin \theta_{i} \simeq \theta_{i}$ and $\cos \theta_{i}$ $\simeq 1,(i=1,2)$ are reasonable.

The equations of motion for vertical and horizontal boom motion are

$$
J_{i+2} \ddot{\theta}_{i+2}+d_{i+2}=K_{i+2} u_{i+2}, \quad(i=1,2)
$$

where $J_{i+2}, d_{i+2}, K_{i+2}$, and $u_{i+2}$ denote the inertia of vertical and horizontal boom motion, the nonlinear force term including disturbance, the plant gain, and the command voltage from the computer, respectively. Equation (1) assumes that inertia of vertical and horizontal boom motion $J_{i+2}$ is constant, and the effect of time variance is included in disturbance $d_{i+2}$.

To compensate for the effect of disturbances, the following disturbance observer ${ }^{(13)}$ is applied to Eq. (1):

$$
u_{i+2}=\frac{J_{i+2}}{K_{i+2}}\left\{v_{i+2}+\frac{\omega_{i+2}}{s+\omega_{i+2}}\left(\frac{K_{i+2}}{J_{i+2}} u_{i+2}-s \dot{\theta}_{i+2}\right)\right\}
$$

where $s, v_{i+2}$, and $\omega_{i+2}$ represent the differential operator, the new virtual control input calculated by the feedback controller, and the cut-off angular frequency of a low-pass filter (LPF), respectively. A block diagram of this control system is shown in Fig. 2. The dynamics of the crane system in the low-frequency range is

$$
\ddot{\theta}_{i+2} \simeq v_{i+2}
$$

On the other hand, the dynamics of the rope-load system can be derived as follows:

$$
\begin{aligned}
& l_{r}\left(1+\theta_{1}^{2}\right) \ddot{\theta}_{1}+l_{r} \theta_{1} \theta_{2} \ddot{\theta}_{2}+l_{b}\left(\cos \theta_{3}-\theta_{1} \sin \theta_{3}\right) \ddot{\theta}_{3}-l_{r} \theta_{2} \ddot{\theta}_{4}+l_{r} \theta_{1}\left(\dot{\theta}_{1}^{2}+\dot{\theta}_{2}^{2}\right) \\
& -l_{b}\left(\sin \theta_{3}+\theta_{1} \cos \theta_{3}\right) \dot{\theta}_{3}^{2}-\left(l_{b} \sin \theta_{3}+l_{r} \theta_{1}\right) \dot{\theta}_{4}^{2}-2 l_{r} \dot{\theta}_{2} \dot{\theta}_{4}+g \theta_{1}=0 \\
& l_{r} \theta_{1} \theta_{2} \ddot{\theta}_{1}+l_{r}\left(1+\theta_{2}^{2}\right) \ddot{\theta}_{2}-l_{b} \theta_{2} \sin \theta_{3} \ddot{\theta}_{3}+\left(l_{b} \sin \theta_{3}+l_{r} \theta_{1}\right) \ddot{\theta}_{4}+l_{r} \theta_{2}\left(\dot{\theta}_{1}^{2}+\dot{\theta}_{2}^{2}\right) \\
& -l_{b} \theta_{2} \cos \theta_{3} \dot{\theta}_{3}^{2}-l_{r} \dot{\theta}_{2} \dot{\theta}_{4}^{2}+2 l_{b} \cos \theta_{3} \dot{\theta}_{3} \dot{\theta}_{4}+2 l_{r} \dot{\theta}_{1} \dot{\theta}_{4}+g \theta_{2}=0
\end{aligned}
$$

where $g$ is the gravitational acceleration. The derivation of Eqs. (4) and (5) is described in Appendix A.

We assume the variables of the crane system and their time derivatives to be represented as follows:

$$
\theta_{n}=\theta_{n f}+x_{n}, \dot{\theta}_{n}=\dot{\theta}_{n f}+\dot{x}_{n}, \ddot{\theta}_{n}=\ddot{\theta}_{n f}+\ddot{x}_{n}, \quad(n=1,2,3,4)
$$

where $\theta_{n f}$ and $\dot{\theta}_{n f}$ are the desired state variables, $\ddot{\theta}_{n f}$ is the desired acceleration, and $x_{n}, \dot{x}_{n}$, and $\ddot{x}_{n}$ are deviations of signals from the desired ones.

We linearize the crane dynamics around the desired position of the boom by assuming that $\theta_{i}$ and $\dot{\theta}_{i}(i=1,2)$ are small, such that $\theta_{i} \theta_{p} \simeq 0, \theta_{i} \dot{\theta}_{p} \simeq 0, \dot{\theta}_{i} \dot{\theta}_{p+2} \simeq 0, \theta_{i} \dot{\theta}_{p+2}^{2} \simeq 0$, and $\theta_{i} \ddot{\theta}_{p+2} \simeq 0(p=1,2)$ are satisfied. In addition, $\dot{\theta}_{p+2}$ is assumed to be small such that $\dot{\theta}_{3} \dot{\theta}_{4} \simeq 0$ and $\dot{\theta}_{p+2}^{2} \simeq 0$ are satisfied. These assumptions lead to the following linear state equation:

$$
\begin{aligned}
\dot{x}_{i} & =A_{i} x_{i}+b_{i} v_{i+2}, \quad(i=1,2) \\
A_{i} & =\left[\begin{array}{cccc}
0 & 0 & 1 & 0 \\
0 & 0 & 0 & 1 \\
-\xi & 0 & 0 & 0 \\
0 & 0 & 0 & 0
\end{array}\right], b_{i}=\left[\begin{array}{c}
0 \\
0 \\
-\gamma_{i} \\
1
\end{array}\right], x_{i}=\left[\begin{array}{c}
x_{i} \\
x_{i+2} \\
\dot{x}_{i} \\
\dot{x}_{i+2}
\end{array}\right]
\end{aligned}
$$

where $\xi=g / l_{r}, \gamma_{1}=l_{b} \cos \theta_{3 f} / l_{r}$, and $\gamma_{2}=l_{b} \sin \theta_{3 f} / l_{r}$.

Hence, we can use $v_{1}$ to control $\theta_{1}$ and $\theta_{3}$, and $v_{2}$ to control $\theta_{2}$ and $\theta_{4}$. Because the structures of the two state equations are the same, we can apply the same controller. 


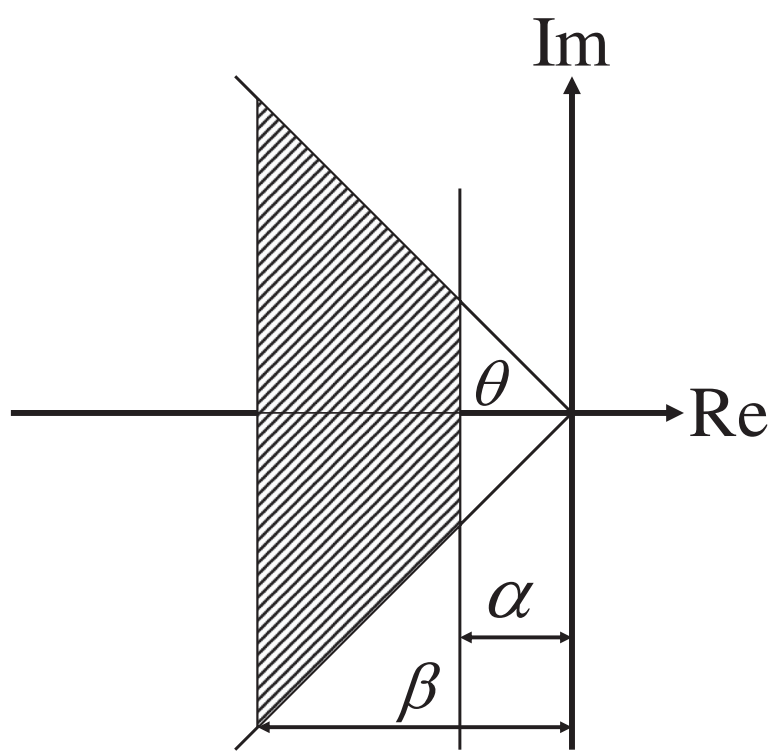

Fig. 3 Stable region

\section{Robust Controller Design}

This section presents a controller for achieving robust performance with respect to rope length variance on the basis of an LMI approach.

\subsection{LMI Representation of Stable Region}

Dynamical system $\dot{x}=A x$ is stable if all eigenvalues of matrix $A$ lie in the left-half of a complex plane. However, considering robustness with respect to variations in matrix $A$ and control performance, control design requires a suitable subregion of the left-half of the plane. To achieve this, Mahmoud and Pascal proposed a method for representing the stable complex region, indicated by the shaded region in Fig. $3^{(14)}$. The condition that all eigenvalues of $A$ lie in the shaded region in Fig. 3 is represented as follows:

$$
\begin{aligned}
& A X+X A^{T}+2 \alpha X<0 \\
& A X+X A^{T}+2 \beta X<0 \\
& {\left[\begin{array}{cc}
\sin \theta\left((A X)+(A X)^{T}\right) & \cos \theta\left((A X)-(A X)^{T}\right) \\
\cos \theta\left((A X)^{T}-(A X)\right) & \sin \theta\left((A X)+(A X)^{T}\right)
\end{array}\right]<0} \\
& X>0
\end{aligned}
$$

where $\theta, \alpha$, and $\beta$ are the positive scalars, and $X$ is the symmetric matrix.

\subsection{State Feedback Control with Integrator}

We consider the following linear control system with an integrator for a rotary crane:

$\dot{x}_{i}^{\prime}=A_{i}^{\prime} x_{i}^{\prime}+b_{i}^{\prime} v_{i+2}, \quad(i=1,2)$

$$
\begin{aligned}
A_{i}^{\prime} & =\left[\begin{array}{ccccc}
0 & 0 & 1 & 0 & 0 \\
0 & 0 & 0 & 1 & 0 \\
-\xi & 0 & 0 & 0 & 0 \\
0 & 0 & 0 & 0 & 0 \\
0 & 1 & 0 & 0 & 0
\end{array}\right], b_{i}^{\prime}=\left[\begin{array}{c}
0 \\
0 \\
-\gamma_{i} \\
1 \\
0
\end{array}\right], x_{i}^{\prime}=\left[\begin{array}{c}
x_{i} \\
x_{i+2} \\
\dot{x}_{i} \\
\dot{x}_{i+2} \\
\int_{0}^{t} x_{i+2} d t
\end{array}\right] \\
v_{i+2} & =k_{i}^{T} x_{i}^{\prime}, \quad k_{i}^{T}=\left[\begin{array}{lllll}
k_{i 0} & k_{i 1} & k_{i 2} & k_{i 3} & K_{I i}
\end{array}\right]
\end{aligned}
$$

The closed-loop system can be represented as follows:

$$
\dot{x}_{i}^{\prime}=\left(A_{i}^{\prime}+b_{i}^{\prime} k_{i}^{T}\right) x_{i}^{\prime}, \quad(i=1,2)
$$


Hence, we determine the controller gains by a pole placement and optimal regulator method.

\subsection{LMI Formulation of Pole Placement Problem}

In this section, we formulate the pole placement problem on the basis of LMI. If all poles of the system in Eq. (10) are located in the region shown in Fig. 3, the system is stable. Thus, the pole placement problem can be represented as finding $X$ and $M_{i}$ such that the following equations are satisfied,

$$
\begin{aligned}
& A_{i}^{\prime} X+X A_{i}^{\prime T}+b_{i}^{\prime} M_{i}+M_{i}^{T} b_{i}^{\prime T}+2 \alpha X<0 \\
& A_{i}^{\prime} X+X A_{i}^{\prime T}+b_{i}^{\prime} M_{i}+M_{i}^{T} b_{i}^{\prime T}+2 \beta X<0 \\
& {\left[\begin{array}{cl}
\sin \theta\left(A_{i}^{\prime} X+X A_{i}^{\prime T}+b_{i}^{\prime} M_{i}+M_{i}^{T} b_{i}^{\prime T}\right) & \cos \theta\left(A_{i}^{\prime} X-X A_{i}^{\prime T}+b_{i}^{\prime} M_{i}-M_{i}^{T} b_{i}^{\prime T}\right) \\
\cos \theta\left(-A_{i}^{\prime} X+X A_{i}^{\prime T}-b_{i}^{\prime} M_{i}+M_{i}^{T} b_{i}^{\prime T}\right) & \sin \theta\left(A_{i}^{\prime} X+X A_{i}^{\prime T}+b_{i}^{\prime} M_{i}+M_{i}^{T} b_{i}^{\prime T}\right)
\end{array}\right]<0} \\
& X>0
\end{aligned}
$$

where $M_{i}=k_{i} X$.

\subsection{LMI Formulation of Optimal Regulator Problem}

We consider the following variable and cost function $J^{(14)}$ :

$J=\frac{1}{2} \int_{0}^{\infty} z_{i}^{T} z_{i} d t, \quad(i=1,2)$

$z_{i}=Q_{i}^{1 / 2} x_{i}+R_{i}^{1 / 2} v_{i+2}$

where $Q_{i} \geq 0$ and $R_{i}>0$.

This problem can be transformed into the problem of finding $X_{2}, Y$, and $k_{i}$ such that $\operatorname{Trace}(Y)$ is minimal in the following constraints:

$$
\begin{aligned}
& {\left[\begin{array}{cc}
\left(A_{i}^{\prime}+b_{i}^{\prime} k_{i}\right) X_{2}+X_{2}\left(A_{i}^{\prime}+b_{i}^{\prime} k_{i}\right)^{T} & 0 \\
0 & -I
\end{array}\right]<0} \\
& {\left[\begin{array}{cc}
Y & \left(Q_{i}^{1 / 2}+R_{i}^{1 / 2} k_{i}\right) X_{2} \\
X_{2}\left(Q_{i}^{1 / 2}+R_{i}^{1 / 2} k_{i}\right)^{T} & X_{2}
\end{array}\right]>0}
\end{aligned}
$$

Setting $M_{i 2}=k_{i} X_{2}$ yields

$$
\begin{aligned}
& {\left[\begin{array}{cc}
\left(A_{i}^{\prime} X_{2}+b_{i}^{\prime} M_{i 2}\right)+\left(X_{2} A_{i}^{\prime T}+M_{i 2}^{T} b_{i}^{\prime T}\right) & 0 \\
0 & -I
\end{array}\right]<0} \\
& {\left[\begin{array}{cc}
Y & \left(Q_{i}^{1 / 2} X_{2}+R_{i}^{1 / 2} M_{i 2}\right) \\
\left(Q_{i}^{1 / 2} X_{2}+R_{i}^{1 / 2} M_{i 2}\right)^{T} & X_{2}
\end{array}\right]>0}
\end{aligned}
$$

Therefore, the optimal regulator problem can be transformed into the new problem of finding $X_{2}, Y$, and $M_{i 2}$ that satisfies the above LMI such that $\operatorname{Trace}(Y)$ is minimal.

\subsection{Controller Design}

In this study, we combine the pole placement and optimal regulator problems expressed using LMI to design the controller as follows:

Setting $X=X_{2}$ and $M_{i}=M_{i 2}(i=1,2)$ yield

$$
\begin{aligned}
& A_{i}^{\prime} X+X A_{i}^{\prime T}+b_{i}^{\prime} M_{i}+M_{i}^{T} b_{i}^{\prime T}+2 \alpha X<0 \\
& A_{i}^{\prime} X+X A_{i}^{\prime T}+b_{i}^{\prime} M_{i}+M_{i}^{T} b_{i}^{\prime T}+2 \beta X<0 \\
& {\left[\begin{array}{cl}
\sin \theta\left(A_{i}^{\prime} X+X A_{i}^{\prime T}+b_{i}^{\prime} M_{i}+M_{i}^{T} b_{i}^{\prime T}\right) & \cos \theta\left(A_{i}^{\prime} X-X A_{i}^{\prime T}+b_{i}^{\prime} M_{i}-M_{i}^{T} b_{i}^{\prime T}\right) \\
\cos \theta\left(-A_{i}^{\prime} X+X A_{i}^{\prime T}-b_{i}^{\prime} M_{i}+M_{i}^{T} b_{i}^{\prime T}\right) & \sin \theta\left(A_{i}^{\prime} X+X A_{i}^{\prime T}+b_{i}^{\prime} M_{i}+M_{i}^{T} b_{i}^{\prime T}\right)
\end{array}\right]<0} \\
& X>0
\end{aligned}
$$

$$
\left[\begin{array}{cc}
Y & \left(Q_{i}^{1 / 2} X+R_{i}^{1 / 2} M_{i}\right) \\
\left(Q_{i}^{1 / 2} X+R_{i}^{1 / 2} M_{i}\right)^{T} & X
\end{array}\right]>0
$$

This problem can be numerically solved by using LMI optimization ${ }^{(15)}$. 


\subsection{Robust Control Performance}

In this section, we demonstrate that if the system in Eq. (10) satisfies the pole placement condition mentioned in $\$ 3.3$ at both ends of the rope length variation region, the system also satisfies the pole placement condition with any rope length within the variation region.

Assuming that the rope length variation region is $l_{r 1} \leq l_{r} \leq l_{r 2}$, we have

$\frac{1}{l_{r}}=\varphi_{1} \frac{1}{l_{r 1}}+\varphi_{2} \frac{1}{l_{r 2}}$

where $\varphi_{1}+\varphi_{2}=1$ and $\varphi_{1}, \varphi_{2}>0$.

The system in Eq. (10) can be represented as follows:

$$
\begin{aligned}
\dot{x}_{i}^{\prime} & =\left(\sum_{p=1}^{2} \varphi_{p} A_{i p}+\sum_{p=1}^{2} \varphi_{p} b_{i p} k_{i}^{T}\right) x_{i}^{\prime}, \quad(i, p=1,2) \\
A_{i 1} & =\left[\begin{array}{ccccc}
0 & 0 & 1 & 0 & 0 \\
0 & 0 & 0 & 1 & 0 \\
-\xi_{1} & 0 & 0 & 0 & 0 \\
0 & 0 & 0 & 0 & 0 \\
0 & 1 & 0 & 0 & 0
\end{array}\right], A_{i 2}=\left[\begin{array}{ccccc}
0 & 0 & 1 & 0 & 0 \\
0 & 0 & 0 & 1 & 0 \\
-\xi_{2} & 0 & 0 & 0 & 0 \\
0 & 0 & 0 & 0 & 0 \\
0 & 1 & 0 & 0 & 0
\end{array}\right], \\
b_{i 1} & =\left[\begin{array}{c}
0 \\
0 \\
-\gamma_{i 1} \\
1 \\
0
\end{array}\right], b_{i 2}=\left[\begin{array}{c}
0 \\
0 \\
-\gamma_{i 2} \\
1 \\
0
\end{array}\right],
\end{aligned}
$$

where $\xi_{1}=g / l_{r 1}, \xi_{2}=g / l_{r 2}, \gamma_{i 1}=l_{b} \cos \theta_{3 f} / l_{r 1}$, and $\gamma_{i 2}=l_{b} \sin \theta_{3 f} / l_{r 2}$.

Therefore, the condition in Eq. (11) can be represented as follows:

$A_{i 1} X+X A_{i 1}^{T}+b_{i 1} M_{i}+M_{i}^{T} b_{i 1}^{T}+2 \alpha X<0$

$A_{i 1} X+X A_{i 1}^{T}+b_{i 1} M_{i}+M_{i}^{T} b_{i 1}^{T}+2 \beta X<0$

$\left[\begin{array}{cc}\sin \theta\left(A_{i 1} X+X A_{i 1}^{T}+b_{i 1} M_{i}+M_{i}^{T} b_{i 1}^{T}\right) & \cos \theta\left(A_{i 1} X-X A_{i 1}^{T}+b_{i 1} M_{i}-M_{i}^{T} b_{i 1}^{T}\right) \\ \cos \theta\left(-A_{i 1} X+X A_{i 1}^{T}-b_{i 1} M_{i}+M_{i}^{T} b_{i 1}^{T}\right) & \sin \theta\left(A_{i 1} X+X A_{i 1}^{T}+b_{i 1} M_{i}+M_{i}^{T} b_{i 1}^{T}\right)\end{array}\right]<0$ $X>0$

$A_{i 2} X+X A_{i 2}^{T}+b_{i 2} M_{i}+M_{i}^{T} b_{i 2}^{T}+2 \alpha X<0$

$A_{i 2} X+X A_{i 2}^{T}+b_{i 2} M_{i}+M_{i}^{T} b_{i 2}^{T}+2 \beta X<0$

$\left[\begin{array}{cc}\sin \theta\left(A_{i 2} X+X A_{i 2}^{T}+b_{i 2} M_{i}+M_{i}^{T} b_{i 2}^{T}\right) & \cos \theta\left(A_{i 2} X-X A_{i 2}^{T}+b_{i 2} M_{i}-M_{i}^{T} b_{i 2}^{T}\right) \\ \cos \theta\left(-A_{i 2} X+X A_{i 2}^{T}-b_{i 2} M_{i}+M_{i}^{T} b_{i 2}^{T}\right) & \sin \theta\left(A_{i 2} X+X A_{i 2}^{T}+b_{i 2} M_{i}+M_{i}^{T} b_{i 2}^{T}\right)\end{array}\right]<0$ $X>0$

Because the coefficient matrices $A_{i}^{\prime}$ and $b_{i}^{\prime}(\mathrm{i}=1,2)$ of the control system in Eq. (10) are linear functions about the term $1 / l_{r}$, the conditions in Eqs. (19) and (20) are also linear functions about the term $1 / l_{r}$. Hence, if there exist $X>0$ and $M_{i}>0$ that satisfy the condition in Eq. (11) for $A_{i}^{\prime}=A_{i p}, b_{i}^{\prime}=b_{i p}(i, p=1,2)$, then $X$ and $M_{i}$ also satisfy the condition in Eq. (11) for $A_{i}^{\prime}=\Sigma_{p=1}^{2} \varphi_{p} A_{i p}, b_{i}^{\prime}=\Sigma_{p=1}^{2} \varphi_{p} b_{i p}\left(\varphi_{p}>0, \varphi_{1}+\varphi_{2}=1\right)$.

Therefore, robust control performance with respect to rope length variance can be achieved.

\section{Results and Discussion}

\subsection{Experimental System}

The experimental system is shown in Fig. 4. Same DC servo motors are used for both vertical and horizontal boom motion. Vertical and horizontal boom angle $\theta_{i+2},(i=1,2)$ is measured by rotary encoders, having angular measurement resolution of $1.8 \times 10^{-3}$ [deg]. 


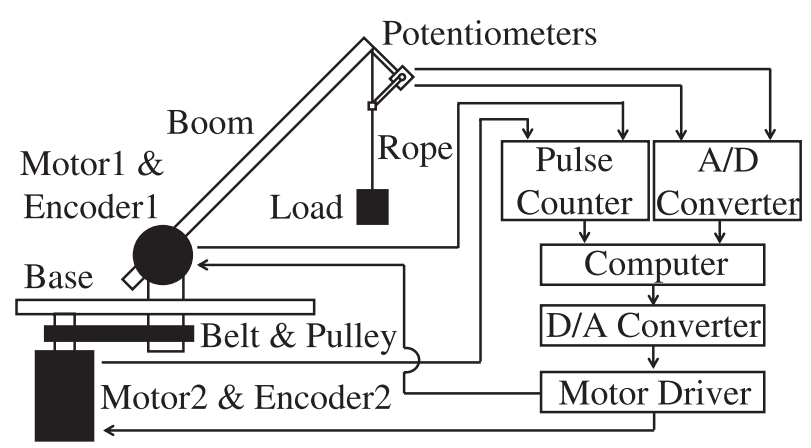

Fig. 4 Experimental system

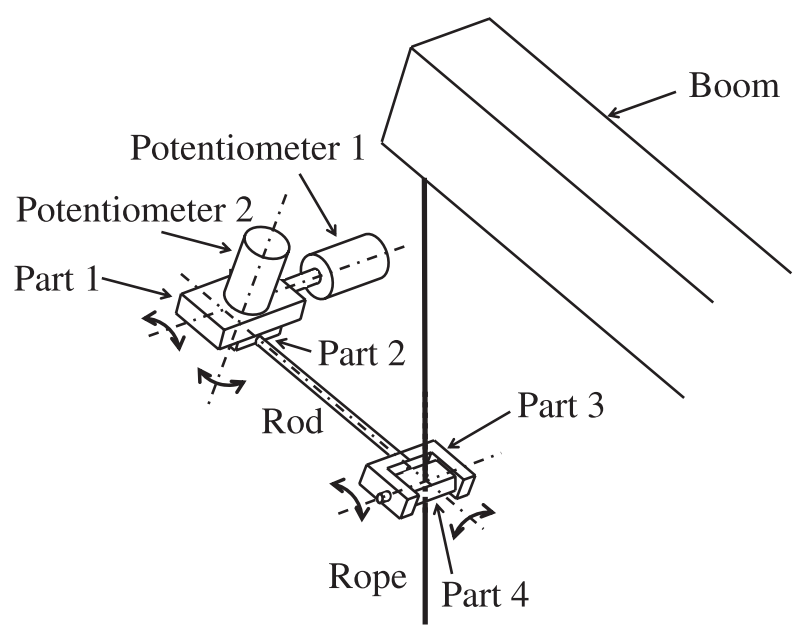

Fig. 5 Sensor system for measuring load sway

Load sway angles $\theta_{1}$ and $\theta_{2}$ are measured by potentiometers 1 and 2 in Fig. 5, respectively. Potentiometer 1 is fixed to the boom. Potentiometer 2 is fixed to part 1 , which rotates around the rotational axis of potentiometer 1 . Part 2 rotates around the rod and the rotational axis of potentiometer 2. Part 3 rotates around the rod, and part 4 rotates around part 3 . Part 4 slides smoothly along the rope. The angular measurement resolution is $6.4 \times 10^{-2}$ [deg]. $\theta_{1}$ and $\theta_{2}$ can be calculated from the output voltage values of potentiometers 1 and 2 , respectively. The parameters of the rotary crane are shown in Table 1.

\subsection{Simulation and Experimental Conditions}

The following cycloid curve is employed to describe the desired angular trajectory of the boom:

$$
r_{q}=\left(\theta_{q f}-\theta_{q 0}\right)\left\{\frac{t}{t_{s}}-\frac{1}{2 \pi} \sin \left(2 \pi \frac{t}{t_{s}}\right)\right\}+\theta_{q 0}, \quad(q=3,4)
$$

where $\theta_{q f}$ is the final angle, $\theta_{q 0}$ is the initial angle, and $t_{s}$ is the settling time. We set $\theta_{30}=$ $30[\mathrm{deg}], \theta_{3 f}=50[\mathrm{deg}], \theta_{40}=0[\mathrm{deg}], \theta_{4 f}=30[\mathrm{deg}]$, and $t_{s}=3[\mathrm{~s}]$. Also, $r_{q}$ is set as $r_{q}=\theta_{q f}$ for $t \in\left(t_{s}, t_{f}\right]$ and $t_{f}=10[\mathrm{~s}]$. The cycloid curve provides zero acceleration at the initial and terminal points, and is widely used in industrial applications.

To determine the controller gains, we set $\theta=78[\mathrm{deg}], \alpha=1$, and $\beta=30$ in Fig. 3 for the robust stable region; we also set $Q_{i}=\operatorname{diag}\{1500,315,1500,315,50\}$ and $R_{i}=1$ in Eq. (12), and considered rope length $l_{r} \in\left[l_{r 1}, l_{r 2}\right]$. The gains were assigned as follows:

$$
\begin{aligned}
& k_{1}=\left[\begin{array}{lllll}
-124 & 39.2 & -0.86 & 25.0 & 23.2
\end{array}\right] \\
& k_{2}=\left[\begin{array}{lllll}
-103 & 38.4 & -0.92 & 24.4 & 22.8
\end{array}\right]
\end{aligned}
$$


Table 1 Parameters of rotary crane

\begin{tabular}{|c|c|c|c|c|c|}
\hline$J_{3}\left[\mathrm{~kg} \cdot \mathrm{m}^{2}\right]$ & 1.02 & $K_{3}[\mathrm{~N} \cdot \mathrm{m} / \mathrm{V}]$ & 2.06 & $J_{4}\left[\mathrm{~kg} \cdot \mathrm{m}^{2}\right]$ & 1.02 \\
\hline$K_{4}[\mathrm{~N} \cdot \mathrm{m} / \mathrm{V}]$ & 2.06 & $L[\mathrm{~m}]$ & 0.65 & $l_{r 1}[\mathrm{~m}]$ & 0.25 \\
\hline$l_{r 2}[\mathrm{~m}]$ & 0.75 & $g\left[\mathrm{~m} / \mathrm{s}^{2}\right]$ & 9.81 & $m[\mathrm{~kg}]$ & 0.20 \\
\hline
\end{tabular}

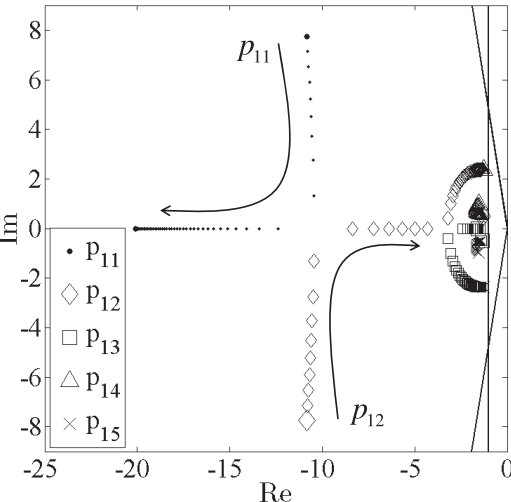

(a) Vertical directional subsystem

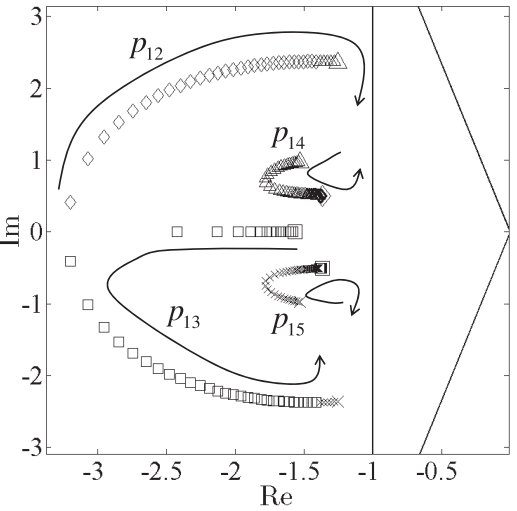

(c) Vertical directional subsystem (Enlarged view)

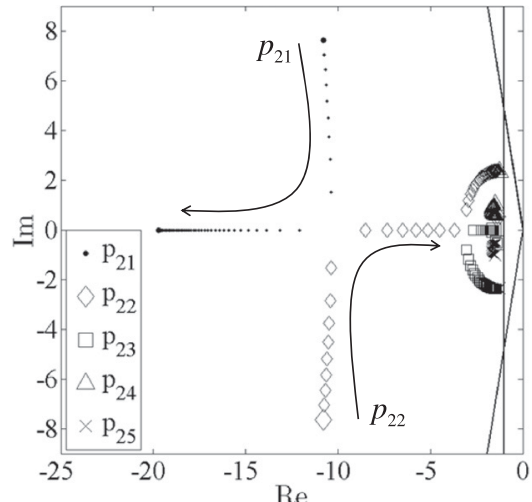

(b) Horizontal directional subsystem

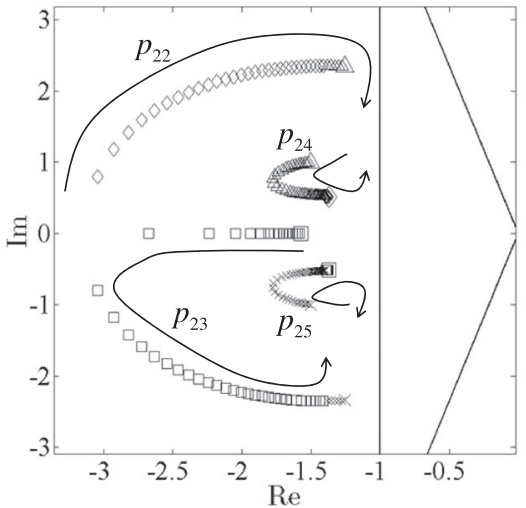

(d) Horizontal directional subsystem (Enlarged view)

Fig. 6 Pole locations (Proposed controller)

In addition, we conducted a simulation to ensure that all poles of the closed-loop system in Eq. (18) lie in the proposed stable region as shown in Fig. 3. The initial poles of the vertical directional subsystem (i.e., under condition $l_{r}=0.25[\mathrm{~m}]$ ) were set to $p_{11}=-10.8+7.74 j[1 / \mathrm{s}]$, $p_{12}=-10.8-7.74 j[1 / \mathrm{s}], p_{13}=-1.57[1 / \mathrm{s}], p_{14}=-1.53+0.97 j[1 / \mathrm{s}]$, and $p_{15}=-1.53-0.97 j[1 / \mathrm{s}]$; those of the horizontal directional subsystem were set to $p_{21}=-10.8+7.63 j[1 / \mathrm{s}], p_{22}=-10.8-$ $7.63 j[1 / \mathrm{s}], p_{23}=-1.57[1 / \mathrm{s}], p_{24}=-1.50+0.99 j[1 / \mathrm{s}]$, and $p_{25}=-1.50-0.99 j[1 / \mathrm{s}]$. The results shown in Fig. 6 confirm that the proposed controller achieves robustness with respect to rope length variance in the interval $\left[l_{r 1}, l_{r 2}\right]$.

\subsection{Simulation and Experimental Results}

To compensate for frictional effects, we conducted a simulation by using the following proportional-derivative (PD) controller with the disturbance observer shown in Fig. 2.

$$
v_{q}=K_{q p} e_{q}+K_{q v} \dot{e}_{q}, \quad e_{q}=r_{q}-\theta_{q}, \quad \dot{e}_{q}=\dot{r}_{q}-\dot{\theta}_{q}, \quad(q=3,4)
$$

where $K_{q p}$ and $K_{q v}$ are the controller gains; they were set to $K_{q p}=50\left[1 / \mathrm{s}^{2}\right]$ and $K_{q v}=10[1 / \mathrm{s}]$, respectively.

In this simulation, the disturbance $d_{i+2}(i=1,2)$ in Eq. (1) includes not only the nonlinear force term but also the following friction term: 


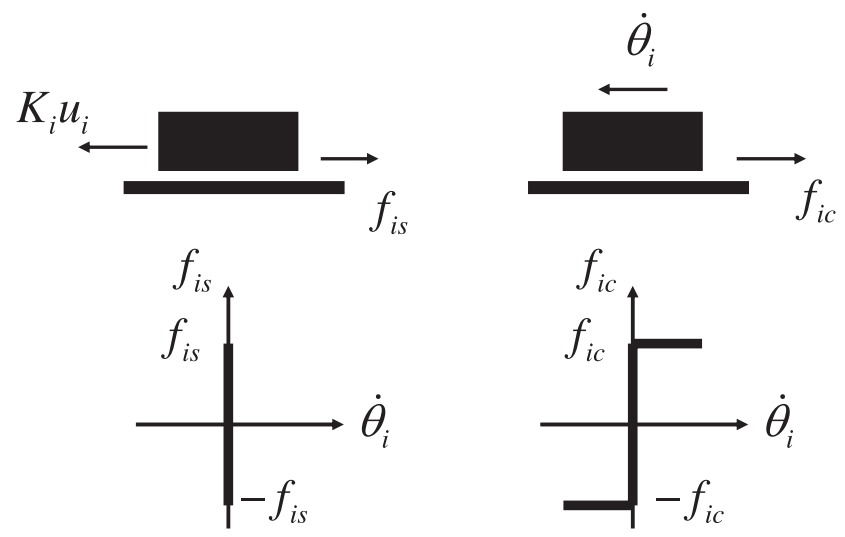

Fig. 7 Friction model

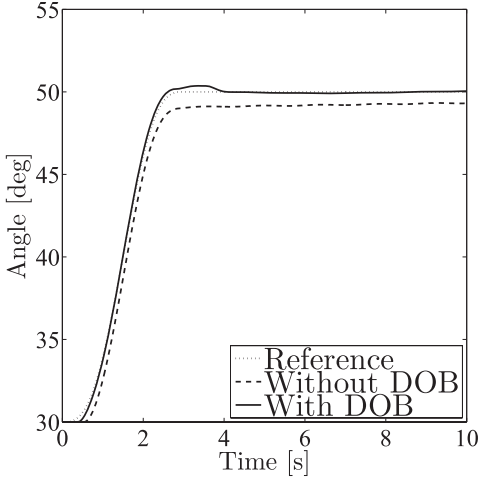

(a) Vertical boom angle $\theta_{3}$

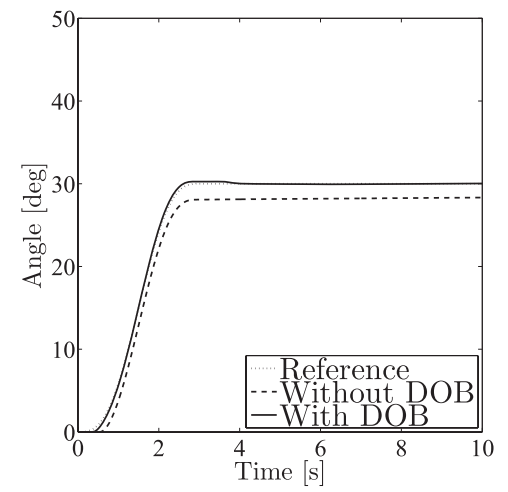

(b) Horizontal boom angle $\theta_{4}$

Fig. 8 Effectiveness of disturbance observer (DOB) (simulation)

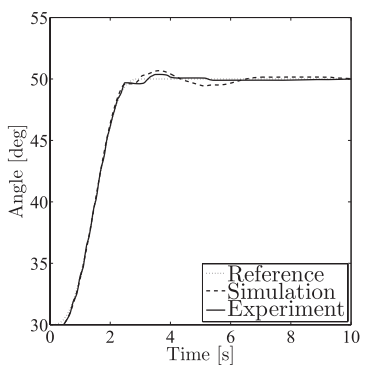

(a) Vertical boom angle $\theta_{3}$

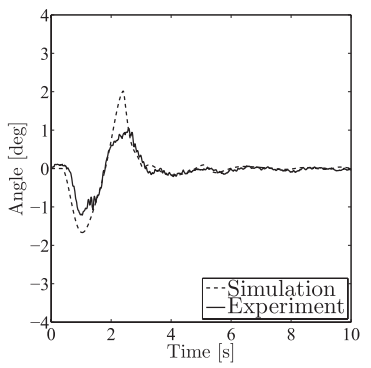

(d) Load sway angle $\theta_{2}$

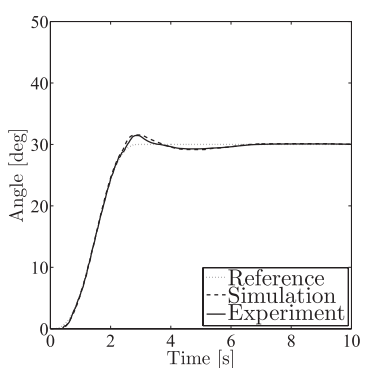

(b) Horizontal boom angle $\theta_{4}$

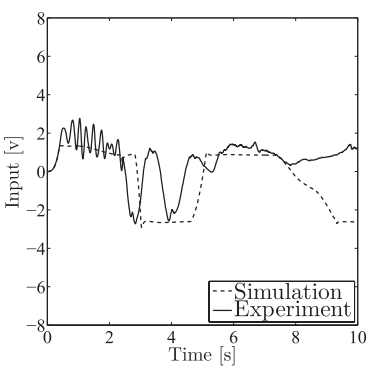

(e) Command voltage $u_{3}$

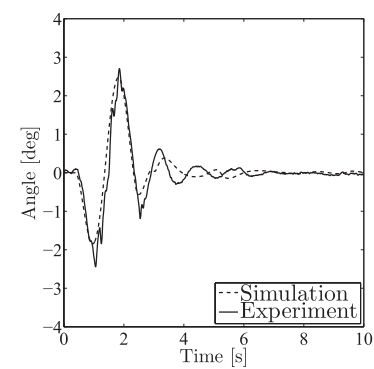

(c) Load sway angle $\theta_{1}$

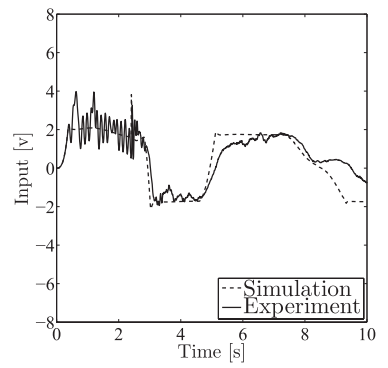

(f) Command voltage $u_{4}$

Fig. 9 Proposed control results $\left(l_{r}=0.50[\mathrm{~m}]\right)$ 


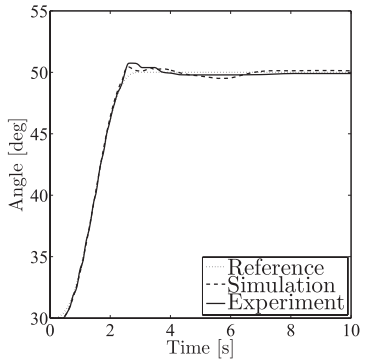

(a) Vertical boom angle $\theta_{3}$

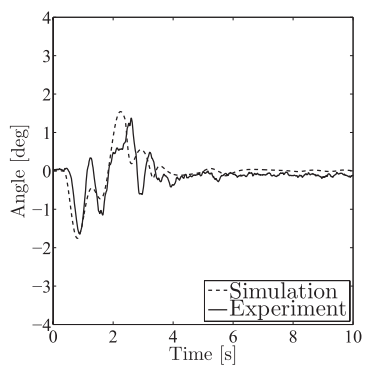

(d) Load sway angle $\theta_{2}$

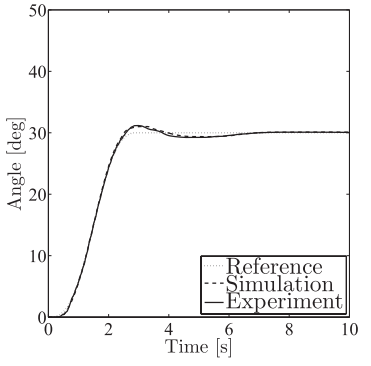

(b) Horizontal boom angle $\theta_{4}$

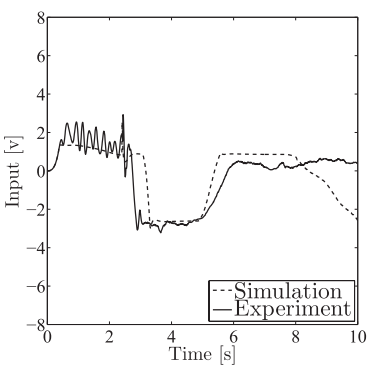

(e) Command voltage $u_{3}$

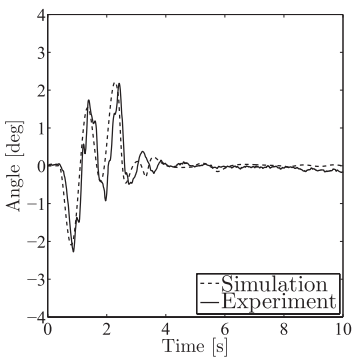

(c) Load sway angle $\theta_{1}$

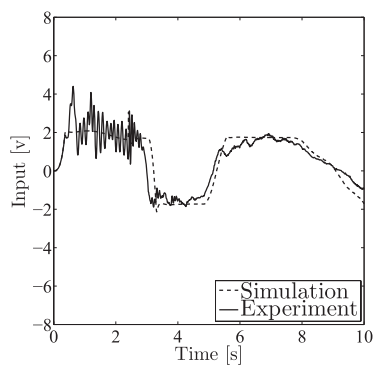

(f) Command voltage $u_{4}$

Fig. 10 Proposed control results $\left(l_{r}=0.25[\mathrm{~m}]\right)$

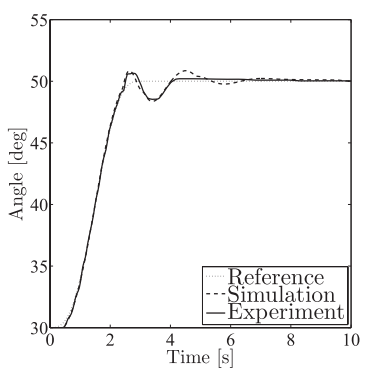

(a) Vertical boom angle $\theta_{3}$

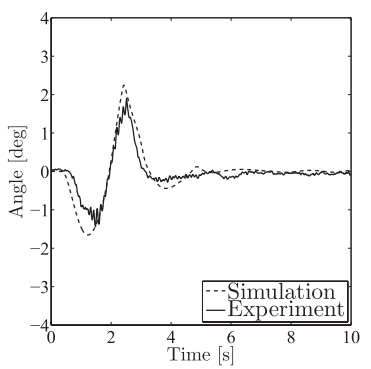

(d) Load sway angle $\theta_{2}$

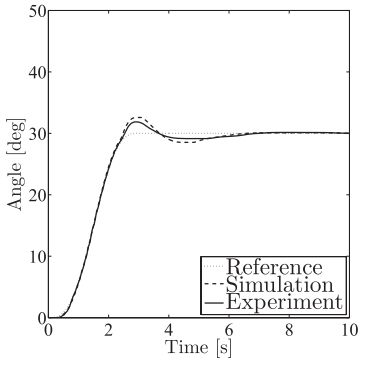

(b) Horizontal boom angle $\theta_{4}$

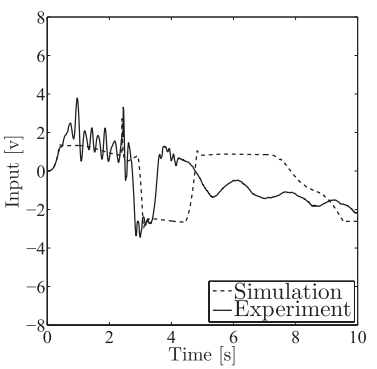

(e) Command voltage $u_{3}$

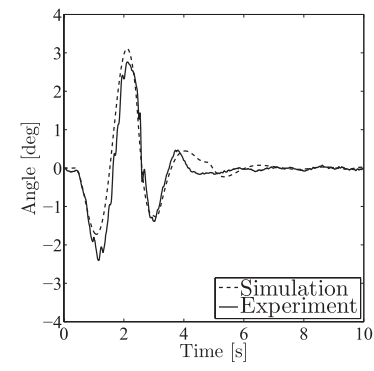

(c) Load sway angle $\theta_{1}$

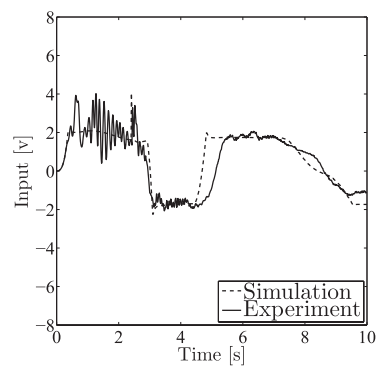

(f) Command voltage $u_{4}$

Fig. 11 Proposed control results $\left(l_{r}=0.75[\mathrm{~m}]\right)$ 


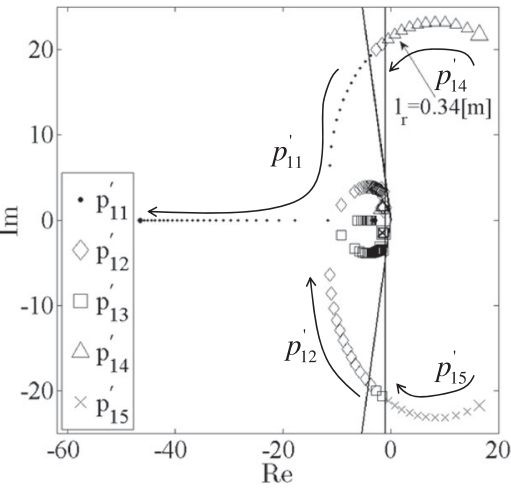

(a) Vertical directional subsystem

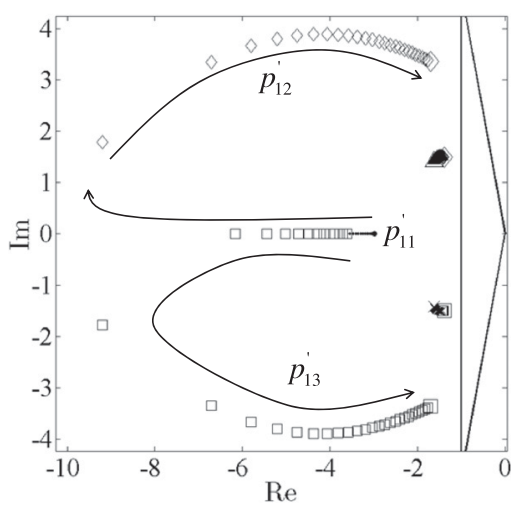

(c) Vertical directional subsystem (Enlarged view)

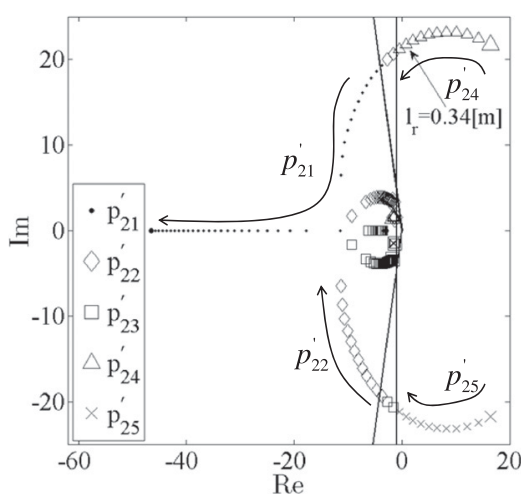

(b) Horizontal directional subsystem

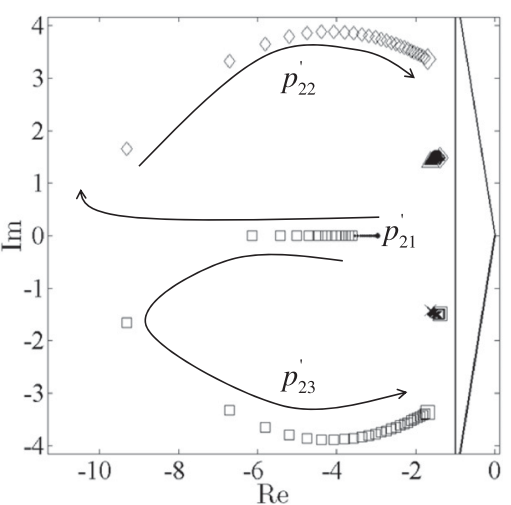

(d) Horizontal directional subsystem (Enlarged view)

Fig. 12 Pole locations (Conventional controller)

$$
f_{q}=C_{q} \dot{\theta}_{q}+f_{q n}, \quad(q=3,4)
$$

where $C_{q}$ denotes the viscous friction coefficient and $f_{q n}$ denotes the static friction or Coulomb friction term. The profile of $f_{q n}$ is shown in Fig. 7, and can be represented as follows:

$$
f_{q n}= \begin{cases}\operatorname{sgn}\left\{K_{q} u_{q}\right\} \min \left\{\left|K_{q} u_{q}\right|, f_{q s}\right\} & \left(\dot{\theta}_{q}=0\right) \\ \operatorname{sgn}\left\{\dot{\theta}_{q}\right\} f_{q c} & \left(\dot{\theta}_{q} \neq 0\right)\end{cases}
$$

where $f_{q s}$ and $f_{q c}$ are the magnitudes of static and Coulomb friction, respectively, and their numerical values are determined experimentally as $C_{3}=1.57[\mathrm{~N} \cdot \mathrm{m} /(\mathrm{rad} / \mathrm{s})], f_{3 s}=3.91[\mathrm{~N} \cdot \mathrm{m}]$, and $f_{3 c}=3.91[\mathrm{~N} \cdot \mathrm{m}] ; C_{4}=1.39[\mathrm{~N} \cdot \mathrm{m} /(\mathrm{rad} / \mathrm{s})], f_{4 s}=3.71[\mathrm{~N} \cdot \mathrm{m}]$, and $f_{4 c}=3.58[\mathrm{~N} \cdot \mathrm{m}]$.

The simulation result is shown in Fig. 8. The cut-off angular frequency for the disturbance observer was set to $\omega=40[\mathrm{rad} / \mathrm{s}]$. All angular velocities were determined by calculating the backward difference between successive positional measurements. The steady-state tracking error shown in Fig. 8 was reduced by using the disturbance observer.

The robustness of the disturbance observer with respect to parameter variance, such as load mass and boom horizontal velocity, was demonstrated by numerical simulation, which is included in Appendix B.

To demonstrate the effectiveness of the proposed LMI-based design, we conducted simulations and experiments and set the rope length to $l_{r}=0.50[\mathrm{~m}]$. The resulting vertical boom angle, horizontal boom angle, command voltages, and load sway angles are shown in Fig. 9. The controller gains obtained in Eq. (22) were used in these results. Although a slight overshoot appears in Figs. 9(a) and (b), load sway angles are suppressed in Figs. 9(c) and (d). 


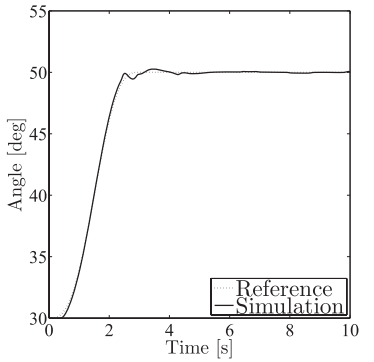

(a) Vertical boom angle $\theta_{3}$

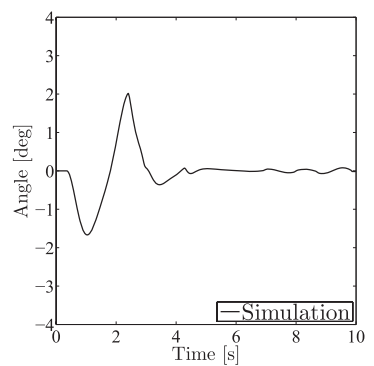

(d) Load sway angle $\theta_{2}$

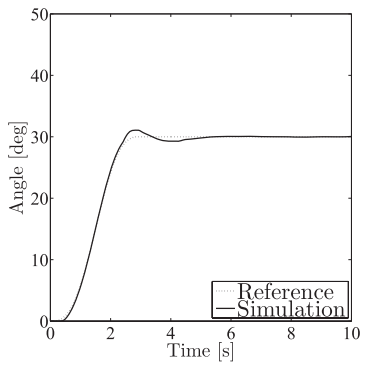

(b) Horizontal boom angle $\theta_{4}$

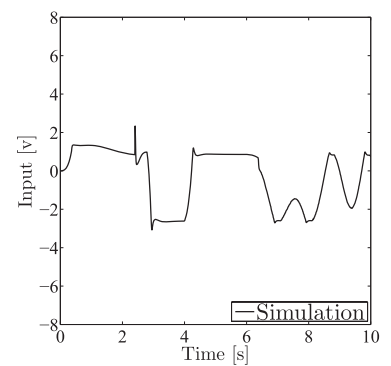

(e) Command voltage $u_{3}$

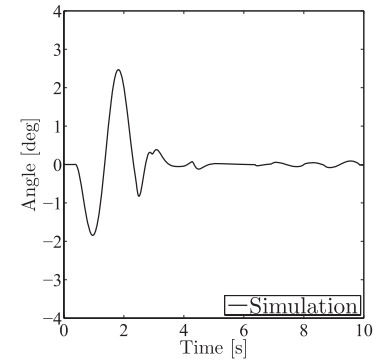

(c) Load sway angle $\theta_{1}$

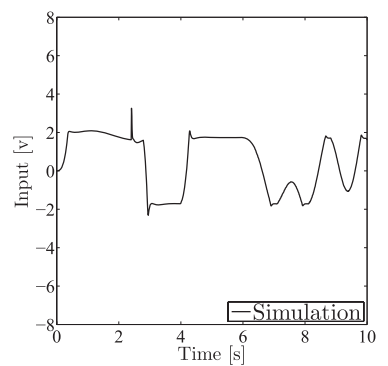

(f) Command voltage $u_{4}$

Fig. 13 Conventional control results $\left(l_{r}=0.50[\mathrm{~m}]\right)$ (simulation)

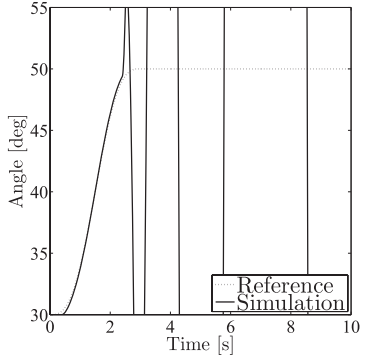

(a) Vertical boom angle $\theta_{3}$

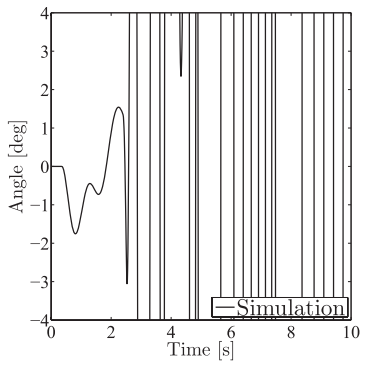

(d) Load sway angle $\theta_{2}$

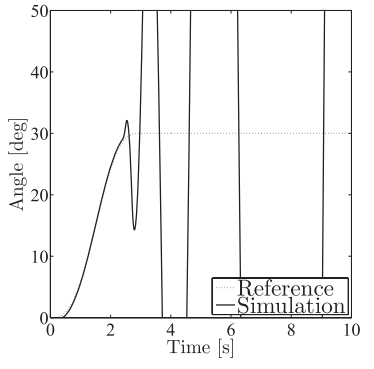

(b) Horizontal boom angle $\theta_{4}$

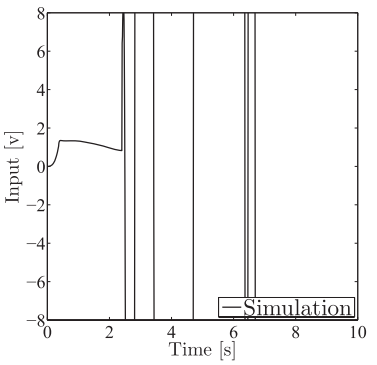

(e) Command voltage $u_{3}$

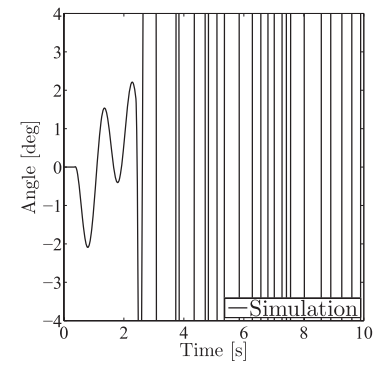

(c) Load sway angle $\theta_{1}$

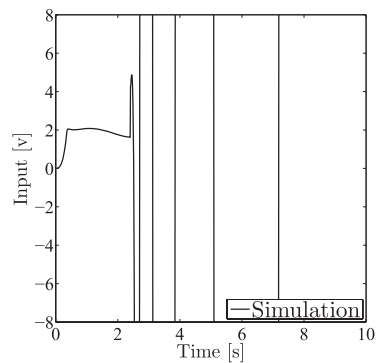

(f) Command voltage $u_{4}$

Fig. 14 Conventional control results $\left(l_{r}=0.25[\mathrm{~m}]\right)$ (simulation) 


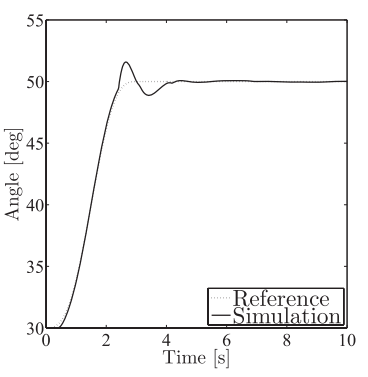

(a) Vertical boom angle $\theta_{3}$

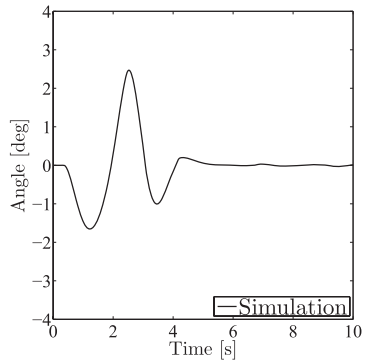

(d) Load sway angle $\theta_{2}$

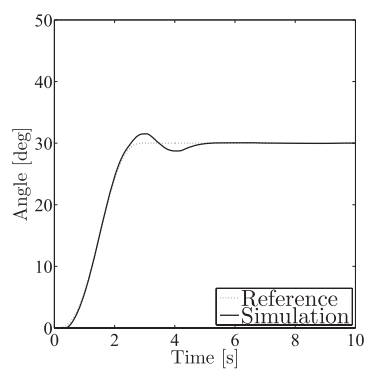

(b) Horizontal boom angle $\theta_{4}$

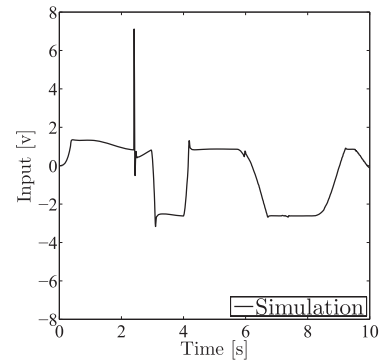

(e) Command voltage $u_{3}$

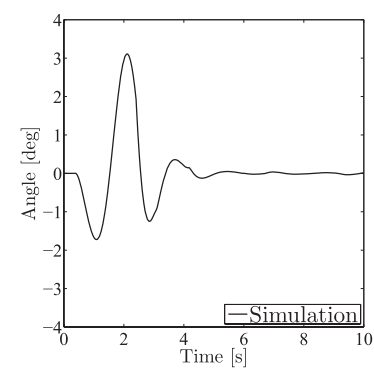

(c) Load sway angle $\theta_{1}$

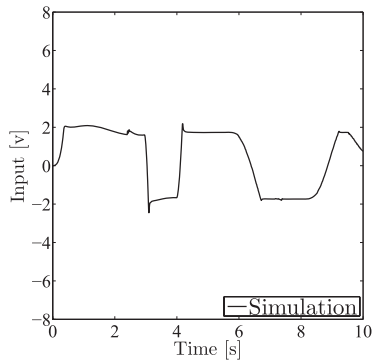

(f) Command voltage $u_{4}$

Fig. 15 Conventional control results $\left(l_{r}=0.75[\mathrm{~m}]\right)$ (simulation)

To demonstrate the robust control performance of the proposed method, we conducted simulations and experiments in which we set the rope length to $l_{r}=0.25[\mathrm{~m}]$ and $l_{r}=0.75[\mathrm{~m}]$ with the same controller gains obtained in Eq. (22). The resulting vertical boom angle, horizontal boom angle, command voltages, and load sway angles are shown in Figs. 10 and 11. The results obtained in these setups were almost the same as those with condition $l_{r}=0.50[\mathrm{~m}]$.

In addition, we conducted a comparative simulation to verify the effectiveness of the proposed method by conventional state feedback with an integrator controller. The controller gains were obtained by a conventional pole placement approach without considering robust control performance (i.e., under condition $\left.l_{r}=0.50[\mathrm{~m}]\right)^{(16)}$.

In this simulation, the dominant poles were set to $-1.5 \pm 1.5 j[1 / \mathrm{s}]$ on the basis of the poles obtained by LMI in $\S 4.2$, and the non-dominant poles were set to $-10 \pm 1.5 j[1 / \mathrm{s}],-10[1 / \mathrm{s}]$ for both vertical and horizontal directional subsystem. The controller gains were designed as follows:

$$
\begin{aligned}
& k_{1}=\left[\begin{array}{lllll}
-181 & 226 & 72.1 & 93.3 & 235
\end{array}\right] \\
& k_{2}=\left[\begin{array}{lllll}
-152 & 226 & 60.5 & 93.3 & 235
\end{array}\right]
\end{aligned}
$$

Pole locations by using controller gains in Eq. (26) are shown in Fig. 12. When $l_{r}=$ $0.25[\mathrm{~m}]$, pole locations are $p_{11}^{\prime}=p_{21}^{\prime}=-2.98[1 / \mathrm{s}], p_{12}^{\prime}=p_{22}^{\prime}=-1.39+1.49[1 / \mathrm{s}], p_{13}^{\prime}=p_{23}^{\prime}=$ $-1.39-1.49[1 / \mathrm{s}], p_{14}^{\prime}=p_{24}^{\prime}=16.5+21.8[1 / \mathrm{s}]$, and $p_{15}^{\prime}=p_{25}^{\prime}=16.5-21.8[1 / \mathrm{s}]$, and include unstable ones. The closed system becomes unstable when $l_{r}=0.34[\mathrm{~m}]$ as shown in Fig. 12.

The simulation results of vertical boom angle, horizontal boom angle, command voltage, and load sway angles are shown in Figs. 13-15. In Figs. 13 and 15, the results obtained were almost the same as those shown in Figs. 9 and 11, respectively. However, in Fig. 14, the rotary crane control system becomes unstable for the rope length of $l_{r}=0.25[\mathrm{~m}]$ when the controller gains in Eq. (26) are used.

These results confirm the effectiveness of the proposed controller for achieving robustness with respect to rope length variance in rotary crane systems.

\section{Conclusion}

This paper proposed a controller for achieving robustness with respect to the rope length variance. First, we derived a linear model of a rotary crane by using a disturbance observer. 


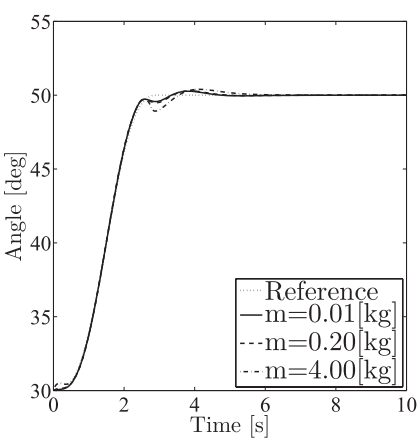

(a) Vertical boom angle $\theta_{3}$

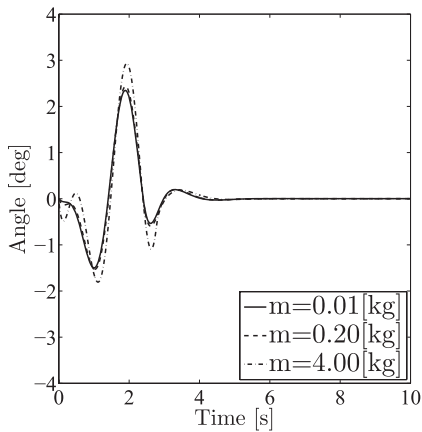

(c) Load sway angle $\theta_{1}$

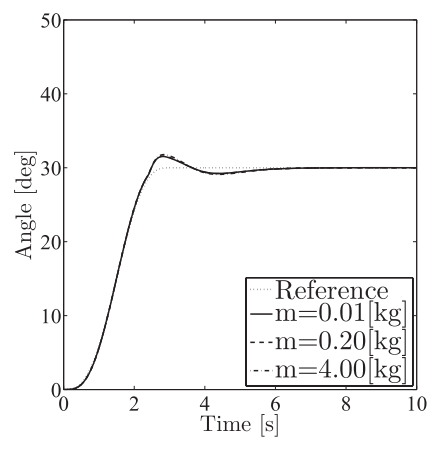

(b) Horizontal boom angle $\theta_{4}$

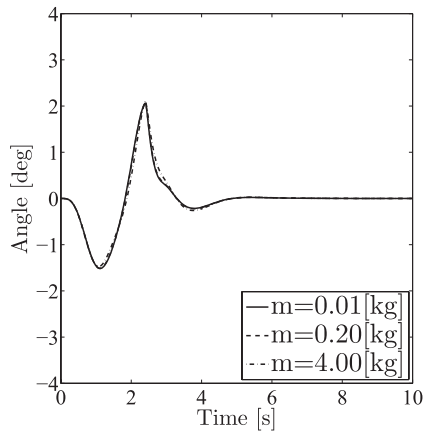

(d) Load sway angle $\theta_{2}$

Fig. B1 Effectiveness of disturbance observer for load mass variance (simulation)

Next, we proposed a controller for achieving robust performance. The controller gains were obtained by solving a pole placement problem and an optimal regulator problem formulated through LMI optimization. The effectiveness of the proposed method was verified by both simulations and experimental results.

\section{Appendix A Derivation of Rope-Load System Dynamics}

This section briefly describes the derivation of the rope-load system dynamics in Eqs. (4) and (5). From Fig. 1, the position of the load is

$$
\left\{\begin{array}{l}
x=l_{b} \sin \theta_{3} \cos \theta_{4}+l_{r} \cos \theta \tan \theta_{1} \cos \theta_{4}-l_{r} \cos \theta \tan \theta_{2} \sin \theta_{4} \\
y=l_{b} \sin \theta_{3} \sin \theta_{4}+l_{r} \cos \theta \tan \theta_{1} \sin \theta_{4}+l_{r} \cos \theta \tan \theta_{2} \cos \theta_{4} \\
z=l_{b} \cos \theta_{3}-l_{r} \cos \theta
\end{array}\right.
$$

where $\theta$ is defined as shown in Fig. 1, and $\tan \theta=\sqrt{\tan ^{2} \theta_{1}+\tan ^{2} \theta_{2}}$ is held. By assuming that $\theta_{1}, \theta_{2}$, and $\theta$ are small so that $\tan \theta_{1} \simeq \theta_{1}, \tan \theta_{2} \simeq \theta_{2}, \cos \theta \simeq 1$ and $\theta=\sqrt{\theta_{1}^{2}+\theta_{2}^{2}}$ are satisfied, the following equations are obtained:

$$
\left\{\begin{array}{l}
x=l_{b} \sin \theta_{3} \cos \theta_{4}+l_{r} \theta_{1} \cos \theta_{4}-l_{r} \theta_{2} \sin \theta_{4} \\
y=l_{b} \sin \theta_{3} \sin \theta_{4}+l_{r} \theta_{1} \sin \theta_{4}+l_{r} \theta_{2} \cos \theta_{4} \\
z=l_{b} \cos \theta_{3}-l_{r} \cos \sqrt{\theta_{1}^{2}+\theta_{2}^{2}}
\end{array}\right.
$$

Noted that the height of the load as well as the potential energy is regarded to be unchanged if $z$ is defined as $z=l_{b} \cos \theta_{3}-l_{r}$ and $\theta_{3}$ is fixed.

The kinetic energy $T$ and potential energy $U$ of the load are defined as follows:

$$
\begin{aligned}
T & =\frac{1}{2} m\left(\dot{x}^{2}+\dot{y}^{2}+\dot{z}^{2}\right) \\
U & =m g z
\end{aligned}
$$

The rope-load system dynamics in Eqs. (4) and (5) can be obtained by using the Lagrange's equations of motion from Eqs. (A2)-(A4). 


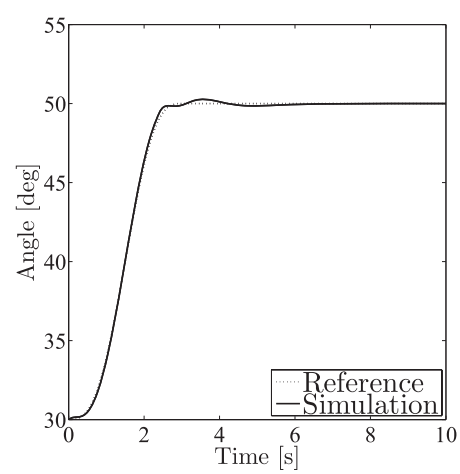

(a) Vertical boom angle $\theta_{3}$

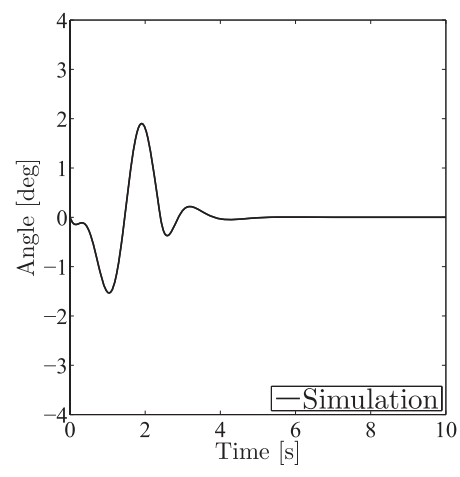

(c) Load sway angle $\theta_{1}$

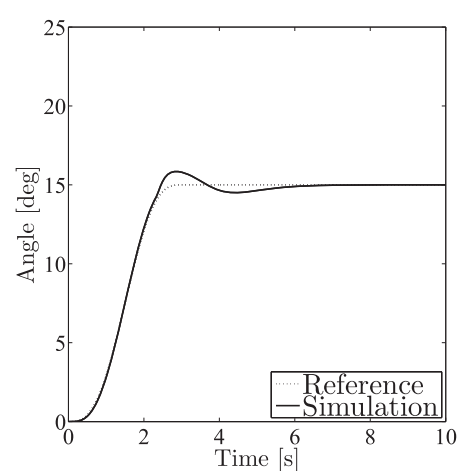

(b) Horizontal boom angle $\theta_{4}$

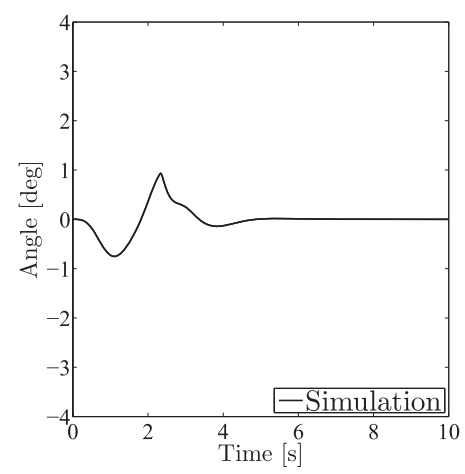

(d) Load sway angle $\theta_{2}$

Fig. B2 Effectiveness of disturbance observer for boom horizontal velocity variance $\left(\theta_{4 f}=15[\mathrm{deg}]\right)$ (simulation)

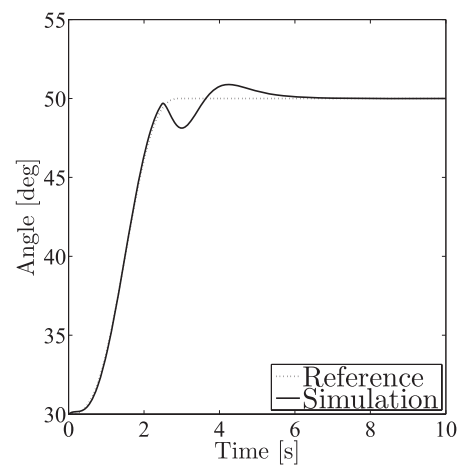

(a) Vertical boom angle $\theta_{3}$

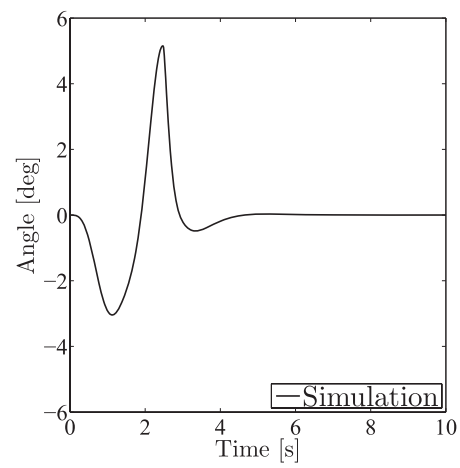

(c) Load sway angle $\theta_{1}$

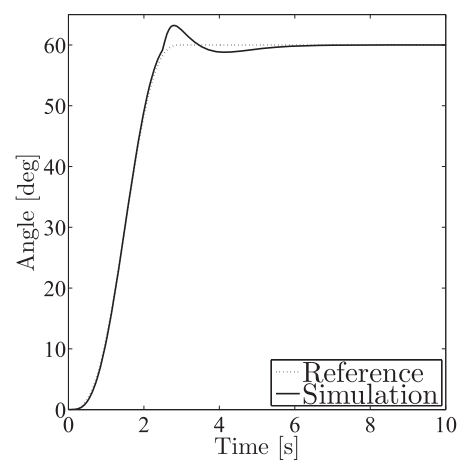

(b) Horizontal boom angle $\theta_{4}$

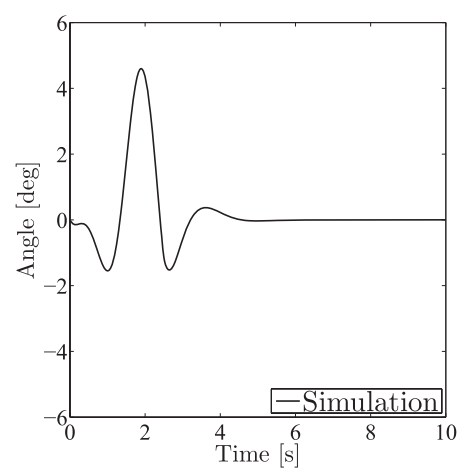

(d) Load sway angle $\theta_{2}$

Fig. B3 Effectiveness of disturbance observer for boom horizontal velocity variance $\left(\theta_{4 f}=60[\mathrm{deg}]\right)$ (simulation) 


\section{Appendix B Simulation for Verifying Robustness of Disturbance Observer}

This section presents simulation results to demonstrate the robustness of the disturbance observer shown in Fig. 2 with respect to parameter variance, such as load mass and boom motion velocity.

Simulation results in Figs. B1-B3 were obtained by the proposed controller without the friction term in Eq. (24). Tracking performance in transient period of $\theta_{3}$ and $\theta_{4}$ were unchanged even when load mass and boom horizontal velocity are largely changed. In addition, load sway frequency was unchanged because the same rope length is assumed. The proposed controller design is concluded to be applicable to practical systems with parameter variance, such as load mass and boom motion velocity.

\section{References}

( 1 ) Sakawa Y, Shindo Y, Optimal control of container cranes, Automatica 1982;18(3):25766.

( 2 ) Sakawa Y, Nakazumi A, Modeling and control of a rotary crane, Transactions of the ASME, Journal of Dynamic Systems, Measurement, and Control 1985;107:200-6.

( 3 ) Terashima K, Shen Y, Yano K, Modeling and optimal control of a rotary crane using the straight transformation method, Control Engineering Practice 2007;15:1179-92.

( 4 ) Lee HH, Modeling and control of a three-dimensional overhead crane, Transactions of the ASME, Journal of Dynamic Systems, Measurement, and Control 1998;120:471-76.

( 5 ) Moustafa KAF, Reference trajectory tracking of overhead cranes, Transactions of the ASME, Journal of Dynamic Systems, Measurement, and Control 2001;123:139-41.

( 6 ) Lee HH, A new motion-planning scheme for overhead cranes with high-speed hoisting, Transactions of the ASME, Journal of Dynamic Systems, Measurement, and Control 2004;126:359-64.

( 7 ) Tsuchiya T, Itoh M, Anti-swing control for rotary cranes, Journal of the Society of Instruments and Control Engineers (In Japanese) 1974;13(10):797-805.

( 8 ) Ohto M, Yasunobu S, A rotary crane control method based on a skillful operator's knowledge, Transactions of the Society of Instruments and Control Engineers (In Japanese) 1997;33(9):923-929.

( 9 ) Kondo R, Takeda T, Taguchi H, Ishibashi J, Sway control for rotary crane based on load swing period, Transactions of the Japan Society of Mechanical Engineers (In Japanese) 2000;67(655):725-731.

(10) Kondo R, Shimahara S, Anti-sway control of a rotary crane via two-mode switching control, Transactions of the Society of Instruments and Control Engineers (In Japanese) 2005;41(4):307-313.

(11) Nakazono K, Ohnishi K, Kinjo H, Yamamoto T, Load swing suppression for rotary crane system using direct gradient descent controller optimized by genetic algorithm, Transactions of the Institute of Systems, Control and Information Engineers (In Japanese) 2009;22(8):303-310.

(12) Nakazono K, Ohnishi K, Kinjo H, Yamamoto T, Vibration control of load for rotary crane system using neural network with GA-based training, Artif. Life Robotics 2008;13:98-101.

(13) Ouyang H, Uchiyama N, Sano S, Suppression of two-dimensional load-sway in rotary crane control using only horizontal boom motion, Journal of System Design and Dynamics 2011;5(4):535-546.

(14) Mahmoud C, Pascal G, $H_{\infty}$ design with pole placement constraints: an LMI approach, IEEE Trans. on Automactic Control 1996;41:358-367.

(15) Pascal G, Arkadi N, Alan J. L, Mahmoud C, LMI control toolbox for use with MATLAB, 1995.

(16) Takagi S, Uchiyama N, Simple robust control of rotary cranes, Transactions of The Japan Society of Mechanical Engineers (in Japanese) 2004;70(690):483-491. 